\title{
Lewis Number Effects in Distributed Flames
}

\author{
A. J. Aspden, M. S. Day and J. B. Bell \\ Lawrence Berkeley National Laboratory, Berkeley, CA 94720, USA
}

\begin{abstract}
Recent computational studies have simulated a mode of distributed premixed combustion where turbulent mixing plays a significant role in the transport of mass and heat near the reaction zone. Under these conditions, molecular transport processes play a correspondingly smaller role. A consequence of burning in this regime is that a change in the gas mixture composition can occur within the flame zone, which modifies the burning rate. The composition depends on the Lewis number (ratio of molecular heat to mass diffusivity), and so the response to the transition to distributed burning will be different for fuels with different Lewis numbers. In this paper, we examine the role of Lewis number on flames in the distributed burning regime. We use high-resolution three-dimensional flame simulations with detailed transport models to explore the turbulent combustion of a range of fuels, specifically lean premixed hydrogen, methane and propane mixtures. The response of the burning rate is found to be more pronounced in hydrogen than in the other fuels.
\end{abstract}




\section{Introduction}

There has been considerable recent interest in fuelflexible lean premixed combustion systems. Burning under lean conditions reduces the exhaust gas temperatures, and consequently, thermal $\mathrm{NO}_{\mathrm{x}}$ emissions. Low-swirl burner technology, introduced by [1] as a tool for studying the fundamental properties of lean premixed turbulent flames has proven to be an effective stabilization mechanism for a wide range of fuels, see [2] and [3]. However, high-swirl injectors, often used in gas turbines, can generate much higher levels of turbulence. For example, [4] have measured turbulent intensities as high as $30 \mathrm{~m} / \mathrm{s}$.

There is little work in the literature that deals with premixed flames under highly turbulent conditions. Peters [5] argues that at sufficiently high Karlovitz numbers, the Kolmogorov scale becomes smaller than the inner layer of the flame, turbulence enhances heat loss to the preheat zone, and the chemistry breaks down. The conclusion is that a premixed flame is unable to survive in the distributed burning regime (or broken reaction zone), which is taken to mean large Karlovitz number $\left(\mathrm{Ka}_{L} \gtrsim 100\right)$, for

$$
\mathrm{Ka}_{L}^{2}=\frac{\check{u}^{3}}{s_{L}^{3}} \frac{l_{L}}{l}
$$

where $\check{u}$ and $l$ are the turbulent rms velocity and integral length scale, respectively, and $s_{L}$ and $l_{L}$ are the flat laminar flame speed and width, respectively.

Aspden et al. [6] considered turbulence-flame interactions in carbon-burning thermonuclear (premixed) flames in type Ia supernovae, where the Lewis number (the ratio of thermal diffusivity to the deficient species diffusivity, Le $=\kappa / \mathcal{D}$ ) was very high. Once the Karlovitz number was sufficiently large, a categorically different mode of burning was observed: a distributed flame. Global extinction was not observed under the conditions studied. Diffusive processes were dominated by turbulent mixing, resulting in an effective unity Lewis number flame. This meant that fuel and temperature were advected materially and mixed by turbulence on time scale faster than diffusive mixing. The burning occurred in a single turbulently-broadened flame, where the local burning rate was much lower than the laminar flame. However, the volume of burning was significantly enhanced, and so the turbulent flame speed was approximately 5 or 6 times faster than the laminar flame speed.

Motivated by [6], two groups began to study distributed flames with terrestrial fuels. Poludnenko [7] reported a distributed flame in a stoichiometric hydrogen-air mixture with simple chemistry, but found that subsonic turbulence could not penetrate the internal flame structure. Aspden et al. [8] also examined premixed hydrogen-air mixtures, but under much leaner conditions, which allowed the flame to become distributed at much lower Mach numbers, and found substantial flame broadening was possible. De- tailed examination of the fuel species scalar fluctuation equation demonstrated that the turbulent mixing was the same order of magnitude as the reaction and molecular dissipation terms, but molecular diffusion remained an important process. However, once again, global extinction was not observed under the conditions studied, and the turbulent flame speed was faster than the laminar flame.

It should be emphasized that there are several differences between the distributed flames mentioned above and the high Karlovitz number flames that have been observed to extinguish experimentally. The distributed flames of [6-8] are confined by periodic lateral boundary conditions, which means there is a pool of hot fluid that is mixed with the fuel by fullydeveloped turbulence. The Bunsen flames of [9-12] and the single vortex-flame interactions of [13, 14] that extinguish at high Karlovitz number, all experience large-scale global mean stretch, which is the likely explanation for the difference in behavior.

In contrast to the very high Lewis number of the supernovae flames of [6], lean premixed hydrogen has a Lewis number of approximately 0.35 to 0.365 for equivalence ratios between $\varphi=0.31$ and 0.4 (the range studied in [8]). Methane and propane flames, each at an equivalence ratio of $\varphi=0.7$, have Lewis numbers of 0.96 and 1.95 , respectively. Following $[6,8]$, this paper examines the effect of Lewis number on distributed flames using high-resolution threedimensional simulations of lean hydrogen, methane and propane flames with detailed chemistry at high Karlovitz number.

A consequence of the effective unity Lewis number due to strong turbulent mixing in the distributed burning regime is that there is a shift in composition through the flame, which changes how intensely the flame burns. Consider the distribution of fuel mole fraction and temperature (each of which can be thought of as an approximate progress variable) through flames with different Lewis numbers. Figure 1 shows the flat laminar flame distribution of fuel mole fraction and temperature for four fuels with different Lewis numbers (hydrogen, methane, propane and carbon in $\mathrm{SNe}$ ), normalized by the fuel mole fraction and the temperature where the mole fraction goes to zero. The cold fuel starts in the top-left of the plot, and burns to hot products in the bottom-right. In the methane flame, the diffusion coefficient of methane is essentially the same as the thermal conductivity, suitably normalized, reflecting the near unity Lewis number of the flame. For this case, the relationship between $T$ and $X\left(\mathrm{CH}_{4}\right)$ is essentially linear over much of the normalized temperature range except for a turnover in the hot region of the flame where there is significant heat release. For the propane flame and the supernova flame, the diffusion coefficient of the fuel is significantly smaller than the thermal conductivity, allowing the fuel to be preferentially heated more rapidly than it diffuses into the flame zone. This results in a shift in the $T$ versus fuel curve in figure 1 so that it lies above the methane curve. Analogously, 
the diffusion coefficient of $\mathrm{H}_{2}$ is higher, resulting enhanced diffusion of fuel into the flame and a shift downward in the $T$ versus $X\left(\mathrm{H}_{2}\right)$ plot. It was shown in $[6,8]$ that as the turbulent intensity was increased, turbulent diffusion began to dominate molecular diffusion and the $T$ versus fuel curves became similar to to the methane versus fuel curve in figure 1 , so we expect that the propane curve will do the same. Since the burning rate increases as fuel concentration and temperature increase, when the flame becomes distributed, it can be expected that the burning rate decreases in the high Lewis number case, and increases in the low Lewis number case.

Despite the decrease in the burning rate in the supernova study, there was significant broadening of the flame, and so the overall flame speed increased, and global extinction was not observed. The low Lewis number of lean hydrogen leads to an increase in the local burning rate as the flame becomes distributed, making global extinction less likely. However, there remains the possibility that the Lewis number can affect the flame response to intense turbulent mixing, and a lean propane flame (Le $>1$ ) may extinguish at high Karlovitz number. Another important difference between terrestrial and supernovae flames is the lack of intermediate species in the latter. It may also be possible to disrupt the chemical pathways in a lean propane flame, which could lead to global extinction.

\section{Computational Methodology}

The simulations presented here are based on a low Mach number formulation of the reacting flow equations. The methodology treats the fluid as a mixture of perfect gases. We use a mixture-averaged model for differential species diffusion and ignore Soret, Dufour, gravity and radiative transport processes. With these assumptions, the low Mach number equations for an open domain are

$$
\begin{aligned}
& \frac{\partial(\rho \boldsymbol{u})}{\partial t}+\nabla \cdot(\rho \boldsymbol{u u})=-\nabla \pi+\nabla \cdot \tau, \\
& \frac{\partial\left(\rho Y_{i}\right)}{\partial t}+\nabla \cdot\left(\rho Y_{i} \boldsymbol{u}\right)=\nabla \cdot\left(\rho \mathcal{D}_{i} \nabla Y_{i}\right)-\dot{\omega}_{i}, \\
& \frac{\partial(\rho h)}{\partial t}+\nabla \cdot(\rho h \boldsymbol{u})=\nabla \cdot\left(\frac{\lambda}{c_{p}} \nabla h\right)+ \\
& \sum_{i} \nabla \cdot\left[h_{i}\left(\rho \mathcal{D}_{i}-\frac{\lambda}{c_{p}}\right) \nabla Y_{i}\right]
\end{aligned}
$$

where $\rho$ is the density, $\boldsymbol{u}$ is the velocity, $Y_{i}$ is the mass fraction of species $i, h$ is the mass-weighted enthalpy of the gas mixture, $T$ is the temperature, and $\dot{\omega}_{i}$ is the net destruction rate for species $i$ due to chemical reactions. Also, $\lambda$ is the thermal conductivity, $\tau$ is the stress tensor, $c_{p}$ is the specific heat of the mixture, and $h_{i}(T)$ and $\mathcal{D}_{i}$ are the enthalpy and species mixtureaveraged diffusion coefficients of species $i$, respectively. These evolution equations are supplemented by an equation of state for a perfect gas mixture.
The basic discretization combines a symmetric operator-split treatment of chemistry and transport with a density-weighted approximate projection method. The projection method incorporates the equation of state by imposing a constraint on the velocity divergence. The resulting integration of the advective terms proceeds on the time scale of the relatively slow advective transport. Faster diffusion and chemistry processes are treated time-implicitly. This integration scheme is embedded in a parallel adaptive mesh refinement algorithm framework based on a hierarchical system of rectangular grid patches. The complete integration algorithm is second-order accurate in space and time, and discretely conserves species mass and enthalpy. The reader is referred to [15] for details of the low Mach number model and its numerical implementation, and to [16] for previous applications of this methodology to the simulation of premixed turbulent flames.

The detailed chemistry and transport models used for the hydrogen flames was taken from the GRIMech 2.11 mechanism [17], which has 9 species and 27 fundamental reactions. The methane mechanism was DRM19 [18], with 19 species and 84 fundamental reactions. The propane mechanism was that of [19], with 71 species and 469 fundamental reactions.

The overall numerical scheme is known to converge with second-order accuracy, and the ability of the scheme to perform direct numerical simulation was examined in [20]. The non-oscillatory finitevolume approach used remains stable even when under-resolved, and so some care is required to ensure that simulations are well-resolved. It was shown in [20] that a marginally resolved viscous simulation has an effective Kolmogorov length scale, $\eta_{e}$ and effective viscosity that are slightly larger than that specified. Dimensional analysis was used to characterize the inviscid extreme, known as Implicit Large Eddy Simulation (ILES), through an analogy with the theory of [21]. From this analysis, an expression for the effective viscosity $\nu_{e}$ was obtained that describes the transition from well-resolved to pure ILES, $\nu_{e}=$ $\nu_{u}+\nu_{\Delta x} \exp \left(-\nu_{u} / 2 \nu_{\Delta x}\right)$, from which the effective Kolmogorov length scale can be derived, where $\nu_{\Delta x}$ is the effective viscosity of a simulation with a cell width $\Delta x$ and zero diffusion, and $\nu_{u}$ is the physical viscosity. In the regime considered here, resolution of the turbulence places more stringent requirements on the simulation than does the chemistry. Detailed simulations, discussed in Aspden et al. [8] show the if $\eta_{e} / \eta<1.5$, which is satisfied for the simulation presented here, then the turbulence is well-resolved.

\section{Turbulent Flame Simulations}

Three-dimensional simulations were conducted of three downward-propagating flames in a high aspect ratio domain (8:1), with periodic lateral boundary conditions, a free-slip base and outflow at the top. A turbulent background velocity field was maintained through a source term in the momentum equations 
following [20], consisting of a superposition of longwavelength Fourier modes. This results in a timedependent zero-mean turbulent velocity field. It was shown in [20] that this approach gives approximately 10 integral length scales across the domain width. An inert calculation was run to establish the turbulence at reduced expense, and the reacting flow simulation was initialized by superimposing a laminar flame solution onto the turbulent velocity field. The base grid in each case was $64 \times 64 \times 256$, with two levels of refinement used once the flame had become established, giving an effective resolution of $256 \times 256 \times 2048$.

The simulations have been configured to have the same Karlovitz and Damköhler number in each case, where freely-propagating values have been used to account for the thermodiffusively unstable nature of the hydrogen flame, see Aspden et al. [22] for details. The domain size in each case is approximately 7.6 times the (freely-propagating) laminar flame width, and the turbulent intensity is approximately 50 times the (freely-propagating) laminar flame speed. The simulation properties are shown in table 1, where the effective Kolmogorov length scale of the simulation is given using the expression derived in [20], which compares well with the estimates of the expected Kolmogorov length scale.

Figure 2 shows two-dimensional slices of density, burning rate and temperature for the three fuels (note only five-eighths of the domain height is shown). The density and temperature are normalized by the laminar flame values, the hydrogen burning rate has been normalized by three times the freely-propagating flame value, and the methane and propane flames have been normalized by twice the laminar value. It is clear that the flames are very similar in structure, and present the characteristics typical of a distributed flame: there is a single turbulentlybroadened flame, where the burning occurs at the high temperature end of the distribution.

There is some variation in the relative widths of the density distributions (figure 2), but they all appear to be distributed flames. Here, we consider two characteristics of distributed flames that were identified in [8]. First, an exponential distribution was found in the probability density function (PDF) of $|\nabla \rho|$. This is characteristic of turbulent scalar mixing, see [2325] for examples. When the flame was not distributed, there was a sharp interface between fuel and products, and the normalized PDF presented more rapid decay than exponential. Second, the local equivalence ratio across the flame was found to be close to constant. This was demonstrated using joint probability density functions of fuel mole fraction and temperature (again, used as a rough measure of progress).

Figure 3 shows PDFs of $|\nabla \rho|$ normalized by the respective standard deviation for the three cases. The data have been conditioned such that only regions where the density is between $10 \%$ and $90 \%$ of the laminar extremes contribute to the PDF. The distributions are all very similar, and close to the exponential distribution shown by the straight dotted line. This is indicative that turbulence is a dominant mixing process, and characteristic of a distributed flame.

Figure 4 shows the JPDF of local equivalence ratio against temperature for each fuel, where the equivalence ratio is defined as $\varphi=\left(4 C_{\mathrm{C}}+C_{\mathrm{H}}\right) / 2 C_{\mathrm{O}}$, where $C_{\mathrm{i}}$ denotes the molar concentration of atom i. Taking the temperature as a measure of progress, the flame burns from left-to-right. The laminar distributions are shown by the solid red lines. In the laminar hydrogen flame, the fuel diffuses downstream leading to a decrease in equivalence ratio. The laminar methane flame experiences little change in equivalence ratio because the fuel Lewis number is close to unity, but there is some effect of hydrogen diffusion. The laminar propane flame becomes richer at first due to oxygen diffusion in the downstream direction. In each case, the turbulent distribution is indeed closer to being constant than the laminar distributions, with much less variation. This is an interesting consequence of burning in the distributed regime. Because turbulent mixing gives rise to essentially the same (turbulent) diffusion coefficient for all species, there is little change in local equivalence ratio. All oxygen-containing molecules are essentially advected in packets with all hydrogen-containing molecules and carbon-containing molecules. This demonstrates further that the flames are in the distributed burning regime. Some variation remains, however, which suggests that species diffusion is still having some effect on the large scales. This observation highlights an interesting difference between the methane flame, which has a Lewis number of approximately 0.96 , and the effective unity Lewis number distributed flame. Individual species have different diffusion coefficients, which is why there can be variation in the local equivalence ratio, as shown by the laminar distribution. In the distributed flame, species diffusion is dictated by the turbulent eddy viscosity, and so is the same for all species.

The main difference between the three cases is in the burning rate. In hydrogen (see figure 2), the burning is both broader and relatively more intense than the other two cases; recall the hydrogen has been normalized by three times the freely-propagating flame value, whereas the other two cases have been normalized by just twice the laminar value, and yet appear to be burning less intensely. This is the main difference between distributed flames at different Lewis numbers. Figure 5 shows a probability density function (PDF) of the burning rate for each case, normalized by the corresponding peak laminar value, and the first moment has been taken to remove effects of non-burning regions near the origin. For methane and propane, the main contribution to the burning is close to the corresponding laminar value, whereas in hydrogen, a significant portion of the burning occurs between 1.5 and 2 times the peak laminar rate.

The different burning rates can be accounted for by considering the distribution of fuel mole fraction against temperature, a joint probability density function (JPDF) of which is shown in figure 6 for the three 
cases. The cold fuel starts in the top-left of the plot, and burns to hot products in the bottom-right, and the solid red line denotes the laminar distribution (recall the laminar profiles were shown in figure 1). In each case there is only a small amount of spread in the turbulent flame distribution, which is close to linear in each case, again consistent with the distributed flames of $[6,8]$. Note in lower turbulence cases, the distribution is close to the laminar distribution, and in moderate turbulence cases, the distribution is broad, due to both differential diffusion and turbulent mixing, see $[6,8]$ for more detail. Because the laminar distribution for hydrogen lies below the linear distribution, the burning becomes enhanced in the distributed burning regime. The methane distribution is close to the laminar flame, which is to be expected because the Lewis number of the flame is close to unity, and so is largely unaffected by the transition to distributed flame. The JPDF of the turbulent propane flame is also close to linear, which suggests turbulence has affected the mixing at low-to-intermediate temperatures. For both methane and propane, the high-temperature end of the distribution (where the burning was observed to take place, see figure 2) is close to the laminar flame distribution, which explains why the burning rate did not vary from the laminar rate significantly (again see 2). Despite these variations with Lewis number, global extinction was not observed in these simulations.

Figure 7 shows the integral of the burning rate as a function of both temperature and fuel mole fraction. Specifically, the rate of destruction of fuel is integrated $Q(T, X)=\int \rho \dot{\omega}_{F} \mathrm{~d} V(T, X)$, where $V(T, X)$ is the volume of fluid with temperature $T$ and fuel mole fraction $X$, and $Q(T, X)$ is then averaged in time. Note the axes are broken to focus on the high temperature regions where the burning takes place. The solid black line denotes the flat laminar flame distribution of fuel mole fraction versus temperature. In the turbulent hydrogen flame, almost all of the burning is seen to take place close to the adiabatic flame temperature, whereas in the other fuels, the burning occurs over a broader range of temperatures, centered around the flat laminar flame distribution. We speculate that the differing behavior is because the turbulent time scale is faster than the burning time scale in hydrogen, and so the fuel is mixed before it burns, and so only occurs at the highest temperature attained. For the other fuels the burning time scale is faster so they can burn over a broader range of temperatures.

\section{Discussion and Conclusions}

The effects of Lewis number on distributed burning have been examined through high-resolution threedimensional simulations of lean premixed hydrogen, methane and propane flames. The simulations were configured to have a Karlovitz number of 410 (where a freely-propagating flame speed and width were used to account for the thermodiffusively-unstable na- ture of the hydrogen flame, following [22]). Twodimensional slices of the density, burning rate and temperature showed that all of the flames appeared very similar in structure, reinforcing the normalization approach of [22]. All three cases were shown to share characteristics typical of distributed flames: the normalized PDFs of $|\nabla \rho|$ were exponential in distribution (indicative of turbulent scalar mixing) and the JPDFs of local equivalence ratio against temperature showed little variation in equivalence ratio (indicative that mixing was dominated by turbulence).

The main effect of Lewis number was found to be in the response of the burning rate. It was shown in [8] that the transition to distributed burning led to an enhanced burning rate in low Lewis number hydrogen flames, and a decrease was reported in high Lewis number carbon-burning supernova flames in [6]. In the present paper, the burning rates of both methane and propane were found to be larger unaffected by the transition to distributed burning, remaining close to the corresponding laminar values. JPDFs of fuel mole fraction against temperature showed that the methane distribution was close to the laminar distribution (as expected). The propane distribution was only affected at low temperatures, where there was little burning, and the distribution at high temperatures was close to the laminar profile. Since the burning rate is a strong function of fuel and temperature, these JPDFs explain the observed burning behavior.

Global extinction was not observed under the conditions investigated, but the present study does not exclude that possibility in other fuels. The distribution of fuel mole fraction against temperature in a flat laminar propane flame is close to the distribution in the distributed flame, and so little change in local burning rate was observed. Another high Lewis number fuel may have a distribution closer to that of the supernova flame. Consequently, the transition to distributed burning may result in a sufficient reduction in local burning rate for global extinction to occur. It should also be noted that the turbulent intensity here was only approximately $10 \mathrm{~m} / \mathrm{s}$, so the possibility remains that even higher levels of turbulence could extinguish the flame.

\section{Acknowledgments}

AJA was supported by a Glenn T. Seaborg Fellowship at LBNL; JBB was supported by the DOE Applied Mathematics Research Program; MSD was supported by the DOE SciDAC Program. Simulations were performed on Lawrencium at LBNL, and on Franklin (under an INCITE award) and Hopper at NERSC. All support is provided by the U.S. Department of Energy under Contract No. DE-AC02$05 \mathrm{CH} 11231$.

\section{References}

[1] B. Bedat, R. K. Cheng, Combust. flame 100 (1995) 485-494. 
[2] D. Littlejohn, R. K. Cheng, Proceedings of the Combustion Institute 31 (2) (2007) 3155 - 3162.

[3] R. K. Cheng, D. Littlejohn, P. A. Strakey, T. Sidwell, Proceedings of the Combustion Institute 32 (2) (2009) $3001-3009$

[4] P. Strakey, T. Sidwell, J. Ontko, Proc. Comb. Inst. 31 (2007) 3173-3180.

[5] N. Peters, Turbulent Combustion, Cambridge University Press, 2000.

[6] A. J. Aspden, J. B. Bell, M. S. Day, S. E. Woosley, M. Zingale, The Astrophysical Journal 689 (2008a) 1173-1185.

[7] A. Y. Poludnenko, E. S. Oran, Combustion and Flame in press (nn) (2010) xx-yy.

[8] A. J. Aspden, M. S. Day, J. B. Bell, Submitted for publication. Available at ccse.lbl.gov.

[9] M. S. Mansour, Y.-C. Chen, N. Peters, in: 24th Symposium (International) on Combustion, 1992, pp. 461468.

[10] Y.-C. Chen, N. Peters, G. A. Schneemann, N. Wruck, U. Renz, M. S. Mansour, Combustion and Flame 107 (3) (1996) 223-244.

[11] Y.-C. Chen, M. S. Mansour, Applied Physics B: Lasers and Optics 64 (5) (1997) 599-605.

[12] M. S. Mansour, N. Peters, Y.-C. Chen, in: International Symposium on Combustion, Vol. 1, Combustion Institute, 1998, pp. 767-774.

[13] W. L. Roberts, J. F. Driscoll, M. C. Drake, L. P. Goss, Combustion and Flame 94 (1) (1993) 58-69.

[14] T. Poinsot, D. Veynante, S. Candel, Journal of Fluid Mechanics 228 (1991) 561-606.

[15] M. S. Day, J. B. Bell, Combust. Theory Modelling 4 (2000) 535-556.

[16] J. B. Bell, M. S. Day, J. F. Grcar, Proc. Combust. Inst. 29 (2002) 1987-1993.

[17] C. T. Bowman, GRI-Mech 2.11, available at http://www.me.berkeley.edu/gri_mech.

[18] A. Kazakov, M. Frenklach, Reduced Reaction Sets based on GRI-Mech 1.2, http://www.me. berkeley. edu/drm/.

[19] C. K. Davis, S. G.and Law, H. Wang, Combustion and Flame 119 (4) (1999) 375 - 399.

[20] A. J. Aspden, N. Nikiforakis, S. B. Dalziel, J. B. Bell, Comm. App. Math. Comput. Sci. 3 (1) (2008b) 101.

[21] A. N. Kolmogorov, Royal Society of London Proceedings Series A 434 (1991) (a) 9-13, and (b) 15-17.

[22] A. J. Aspden, M. S. Day, J. B. Bell, Submitted for publication in the Combustion Institute. Available at ccse.lbl.gov.

[23] J. P. Gollub, J. Clarke, M. Gharib, B. Lane, O. N. Mesquita, Phys. Rev. Lett. 67 (25) (1991) 3507-3510.

[24] Jayesh, Z. Warhaft, Phys. Rev. Lett. 67 (25) (1991) 3503-3506.

[25] S. T. Thoroddsen, C. W. Van Atta, Journal of Fluid Mechanics 244 (1992) 547-566 


\begin{tabular}{|l|c|c|c|}
\hline Case & $\mathrm{H}_{2}$ & $\mathrm{CH}_{4}$ & $\mathrm{C}_{3} \mathrm{H}_{8}$ \\
\hline$\varphi$ & 0.40 & 0.70 & 0.70 \\
$s_{L}(\mathrm{~m} / \mathrm{s})$ & 0.47 & 0.205 & 0.195 \\
$l_{L}(\mathrm{~mm})$ & 0.41 & 0.60 & 0.60 \\
\hline$\check{u}(\mathrm{~m} / \mathrm{s})$ & 23.9 & 10.3 & 9.8 \\
$l(\mathrm{~mm})$ & 0.314 & 0.46 & 0.45 \\
$L(\mathrm{~mm})$ & 3.14 & 4.6 & 4.5 \\
$\Delta x(\mu \mathrm{m})$ & 13 & 18 & 18 \\
$\eta(\mu \mathrm{m})$ & 3.7 & 7.0 & 7.2 \\
$\eta_{e}(\mu \mathrm{m})$ & 5.1 & 8.8 & 8.8 \\
\hline
\end{tabular}

Table 1: Simulation properties. 


\section{List of Figure Captions}

Figure 1. ( $52=115$ words) Distribution of normalized temperature and fuel mole fraction for four fuels with different Lewis numbers.

Figure 2. ( $75=330$ words double column $)$ Two-dimensional slices of density, burning rate and temperature for hydrogen, methane and propane, respectively. Note only five-eighths of the domain height is shown. The density and temperature are normalized by the laminar flame values, the hydrogen burning rate has been normalized by three times the laminar value, and the methane and propane flames have been normalized by twice the laminar value.

Figure 3. ( $53=117$ words $)$ Probability density functions of $|\nabla \rho|$.

Figure 4. (154 = 339 words) JPDFs of local equivalence ratio against temperature.

Figure 5. $(57=126$ words $)$ Normalized first moment of the probability density function of normalized burning rate.

Figure 6. (154 = 339 words $)$ JPDFs of fuel mole fraction against temperature.

Figure 7. (154 = 339 words $)$ Integrated fuel consumption rate as a function of fuel mole fraction and temperature.

Total words from text: $4 * 900+346(157 \mathrm{~mm})=3946$ words

Total words from figures: 1705 words

Words from table: $46=102$ words

Total words: 5753 


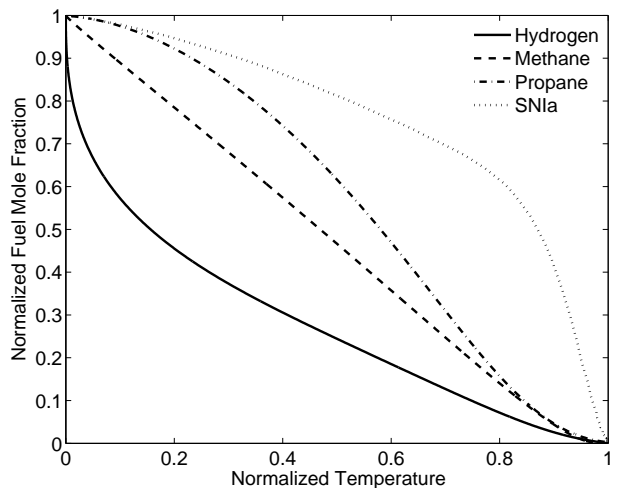

Fig. 1: Distribution of normalized temperature and fuel mole fraction for four fuels with different Lewis numbers. 


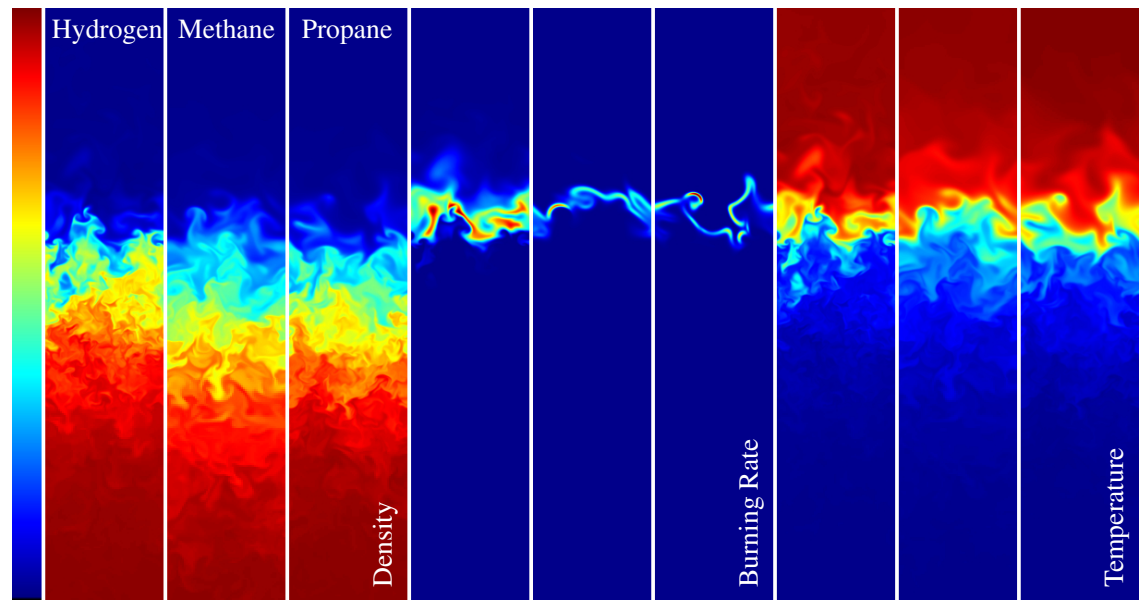

Fig. 2: Two-dimensional slices of density, burning rate and temperature for hydrogen, methane and propane, respectively. Note only five-eighths of the domain height is shown. The density and temperature are normalized by the laminar flame values, the hydrogen burning rate has been normalized by three times the laminar value, and the methane and propane flames have been normalized by twice the laminar value. 


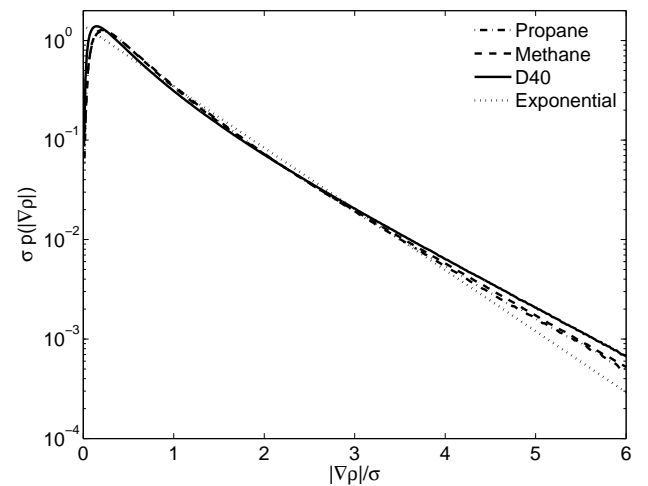

Fig. 3: Probability density functions of $|\nabla \rho|$. 

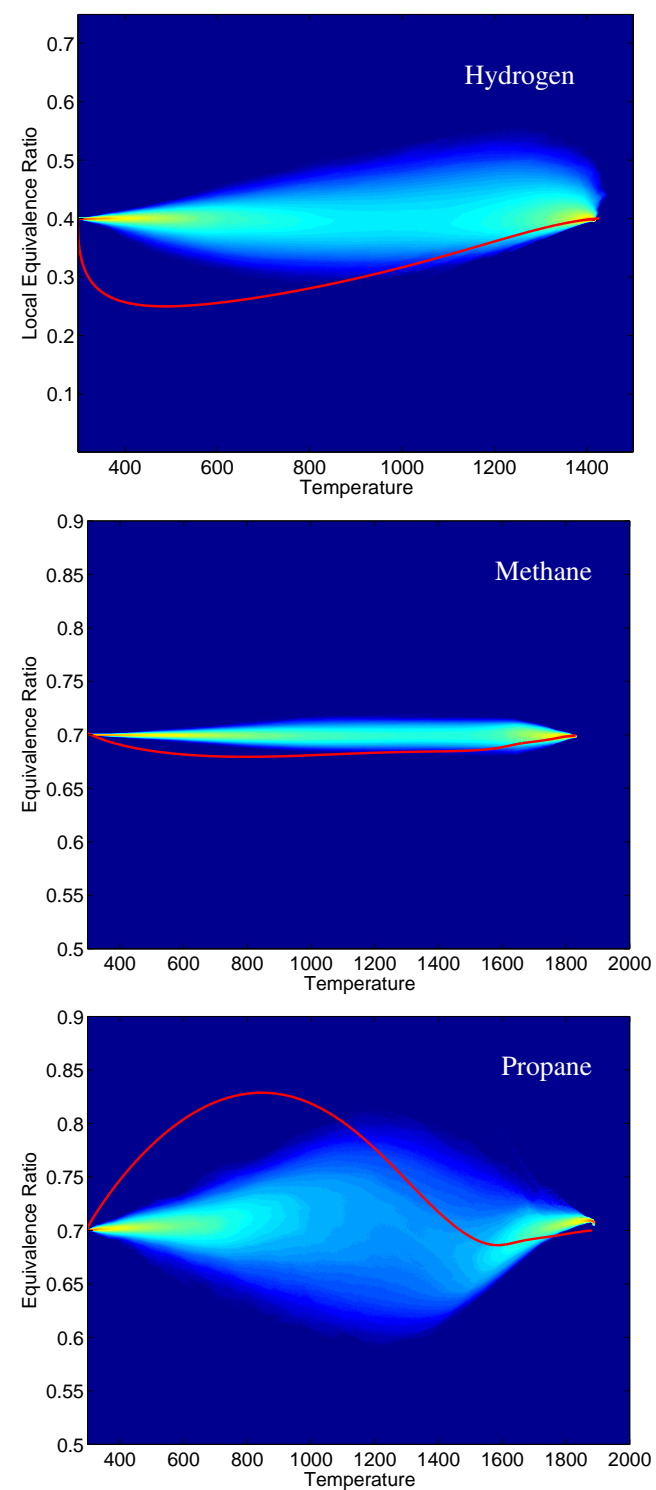

Fig. 4: JPDFs of local equivalence ratio against temperature. 


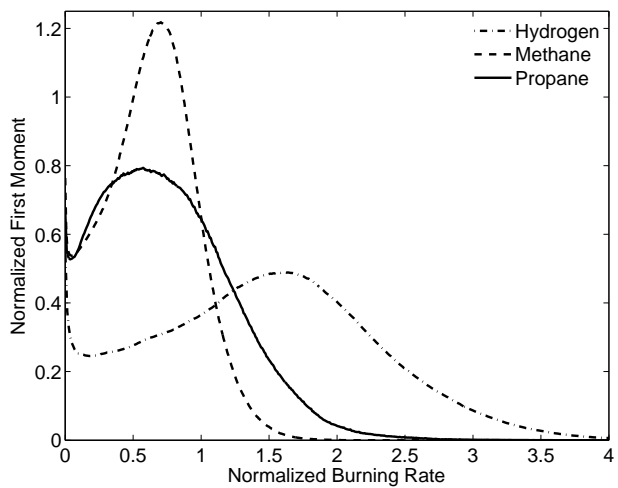

Fig. 5: Normalized first moment of the probability density function of normalized burning rate. 

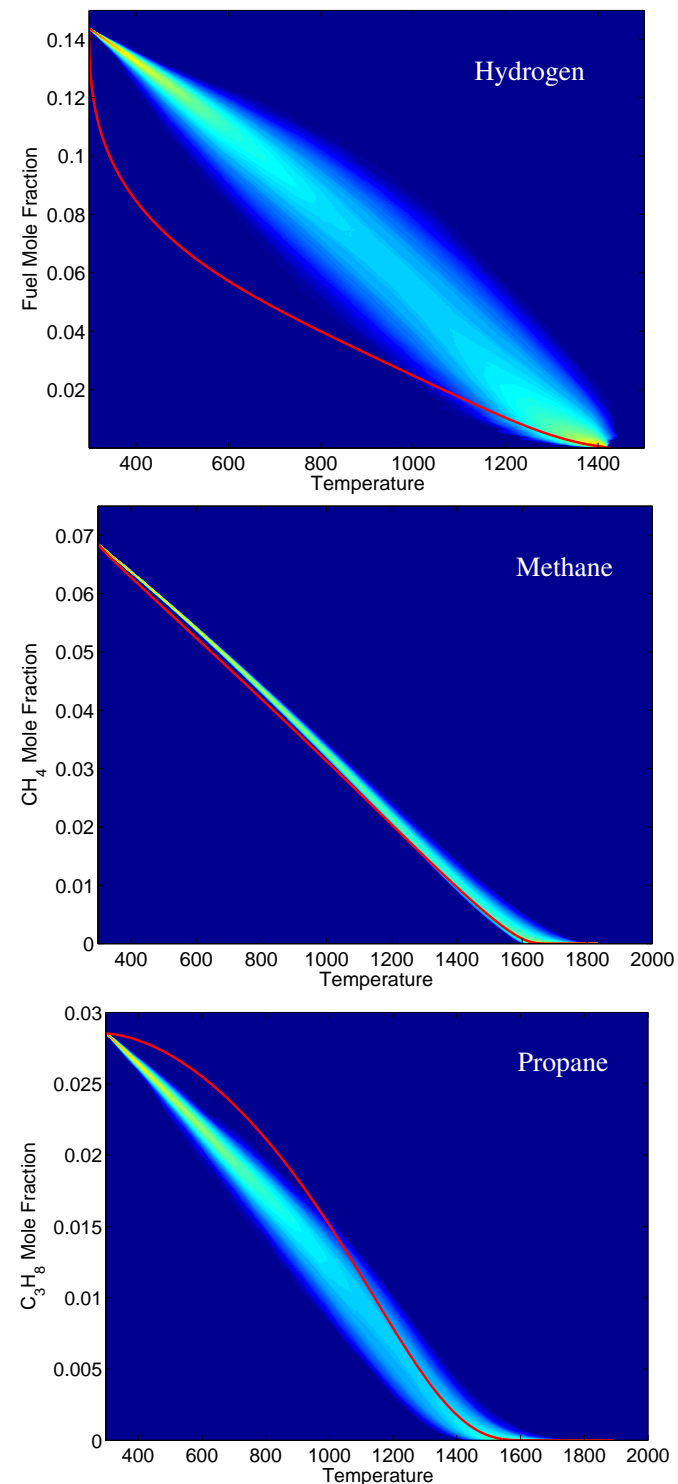

Fig. 6: JPDFs of fuel mole fraction against temperature. 

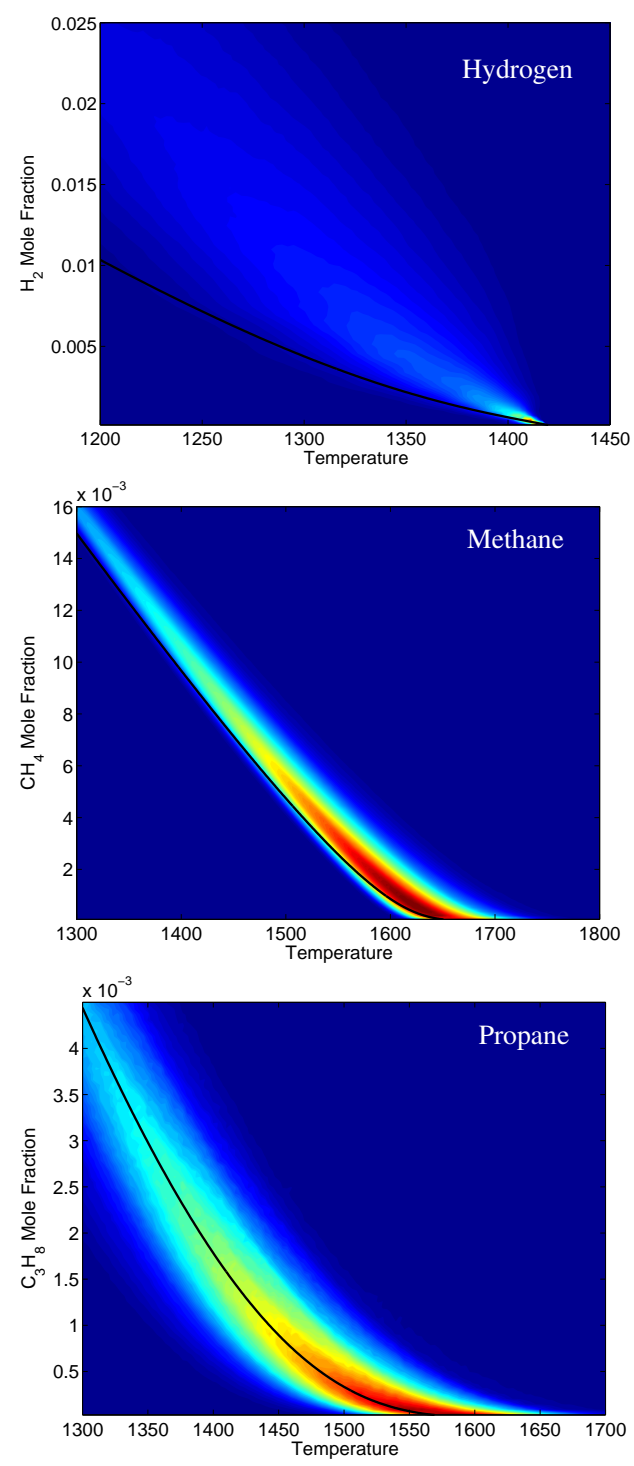

Fig. 7: Integrated fuel consumption rate as a function of fuel mole fraction and temperature. 